\title{
POINT OF CARE CREATININE TESTING IN DIAGNOSTIC IMAGING: A FEASIBILITY STUDY WITHIN THE OUTPATIENT COMPUTED TOMOGRAPHY SETTING.
}

Beverly Snaith, ${ }^{1,2}$ Martine A Harris, ${ }^{1}$ Bethany Shinkins, ${ }^{3}$ Michael Messenger, ${ }^{3}$ Andrew Lewington, ${ }^{4}$ Marieke Jordaan, ${ }^{1}$ Nicholas Spencer ${ }^{1}$

1. Mid Yorkshire NHS Hospitals Trust, Wakefield, UK

2. University of Bradford, Bradford, UK

3. University of Leeds, Leeds, UK

4. Leeds Teaching Hospitals NHS Trust, Leeds, UK

Correspondence to: Professor Beverly Snaith

Address: Faculty of Health Studies, University of Bradford, Richmond Road, Bradford, BD7 1DP

Tel: +44 1274232175

e-Mail: $\underline{\text { b.snaith@bradford.ac.uk }}$

\section{INTRODUCTION ${ }^{1}$}

With imaging demand ever increasing and the expectation for rapid access to imaging growing, opportunities to streamline processes are of interest to both health professionals and administrators. As with all investigations, radiologists and radiographers retain the responsibility to ensure that patient safety is not compromised as a result of quicker turnaround, both from a radiation perspective

$\begin{array}{ll}\text { Abbreviations } & \\ \text { CKD-EPI } & \text { Chronic kidney disease - epidemiology collaboration equation } \\ \text { CSW } & \text { Clinical Support Worker } \\ \text { eGFR } & \text { Estimated glomerular filtration rate } \\ \text { PoC } & \text { Point of Care } \\ \text { PC-AKI } & \text { Post contrast acute kidney injury } \\ \text { QC } & \text { Quality control } \\ \text { SCr } & \text { Serum creatinine } \\ \text { WBCr } & \text { Whole blood creatinine }\end{array}$


and drug administration. With computed tomography (CT) being one of the highest demand areas, contrast administration safety is one key consideration [1]. Although the risks associated with the use of low and iso-osmolar iodinated contrast media are considered marginal [2-8], there is a recognised causal link. The development of post contrast acute kidney injury (PC-AKI), defined as, an increase in serum creatinine of 1.5 times baseline, within 48-72 hours of intravascular administration of a contrast agent [9], is of particular relevance to patients with pre-existing kidney disease $[10,11]$. Despite the ongoing controversy, international guidelines still recommend the screening and risk stratification of patients prior to intravascular contrast administration [9,12-14].

Although the screening of kidney function by blood test is only advocated in those patients with risk factors, many organisations undertake a blanket screening or 'test all' strategy [15]. Within the outpatient radiology setting, Point of Care (PoC) blood testing has been suggested as a mechanism for kidney function testing prior to intravascular iodinated contrast media administration and these devices have been found to be a valid alternative to laboratory testing [16-25]. Although theoretically rapid laboratory result turnaround is possible, this depends on local provision and in practice is usually more than one hour and may not complement CT operations. PoC testing, therefore potentially offers opportunities to reduce unnecessary investigations, reduce patient waits, speed up medical decisionmaking and can promote improved operational efficiency and patient safety $[1,16]$. PoC analysis can be undertaken using a portable hand-held device. However, a range of alternatives are available on the market including benchtop (mini-lab/blood gas) analysers [25]. These allow testing in the radiology department immediately prior to the examination with the process owned by radiology, rather than the hospital laboratory. The benefits of PoC have been considered to be greatest where a screening questionnaire is used to identify patients with co-morbidities which may impair kidney function $[23,24,26]$. However, evaluation of the implementation of such initiatives within UK NHS clinical pathways is limited $[15,25]$.

This feasibility study has evaluated the viability of implementing screening procedures in conjunction with PoC creatinine testing in an outpatient CT service. The objectives included: assessment of individual patient risk factors; the availability of baseline pre-scan day blood test (as mandated locally); comparison of baseline and scan-day kidney function using estimated glomerular filtration rate (eGFR) categorisation; the technical practicalities associated with PoC testing, including failure rates; the clinical concordance of the PoC test against the laboratory gold standard; comparison of serum creatinine $(\mathrm{SCr})$ result pre and post-contrast administration to identify potential PC-AKI. 


\section{MATERIALS AND METHODS}

\section{Study setting}

This study was a prospective single centre (two UK acute hospital sites) feasibility study of PoC kidney function testing within CT.

\section{Study population}

Over an eight-week period between February and April 2017 consecutive adult outpatients ( $\geq 18$ years) attending for a contrast-enhanced CT scan were approached (CT pathway including study specific procedures see Supplementary Figure 1). In advance, patients identified as potentially eligible from the radiology information system by a member of the research team, had been sent a study participant information sheet prior to their appointment. No upper age limit was adopted, but pregnant females and those unable to consent were excluded. As per local protocol at the time of the study, patients with a baseline eGFR below $60 \mathrm{~mL} / \mathrm{min} / 1.73 \mathrm{~m}^{2}$ followed the standard risk reduction regimen of oral hydration ( $1 \mathrm{~L}$ at home) prior to the scan appointment, and all patients were advised to maintain hydration following discharge. Those who declined to participate proceeded straight to scan and followed the standard CT pathway.

\section{Study procedures}

Following written consent, all participants completed a screening questionnaire based on previous studies $[23,24,27-29]$, as is the local standard of care. The questionnaire sought details of known kidney disease, diabetes, heart failure, hypertension, plus history of current/recent acute illness (in the last week, defined as diarrhoea and vomiting; flu; infection requiring antibiotic treatment or acute hospital admission). For the purposes of this feasibility study, the PoC test was performed as an adjunct to the local standard laboratory results (SCr and eGFR), which should have been obtained less than 3 months prior to the CT appointment, to comply with international guidelines [9]. Participants consented to have an additional PoC whole blood creatinine ( $\mathrm{WBCr}$ ) test and a standard laboratory $\mathrm{SCr}$ test performed on the day of the CT appointment. Contrast CT scan procedures followed standard clinical practice, utilising a standard or weight-based volume of intravenous contrast (iohexol; Omnipaque 350; GE Healthcare, Cork, Ireland) appropriate to the anatomy under investigation. All participants were also invited to attend for follow-up bloods tests analysed with the PoC device and in the hospital laboratory at 48-72 hours. PC-AKI was defined as an increase in baseline creatinine level of more than $50 \%$ ( $\geq 1.5$ times baseline) [9]. Additionally we reviewed all cases where there was a relative increase in $\mathrm{WBCr}$ greater than $25 \%$ above baseline, enabling us to explore the characteristics 
of any patient with acute deterioration in kidney function. Where appropriate, individuals meeting this lower threshold were reviewed by the medical team prior to discharge on the day of follow up.

\section{Blood sampling}

All patients were cannulated by a CT radiographer or clinical support worker (CSW) following local standard operating procedures and two whole blood samples were collected. One sample (SMonovette Lithium Heparin $2.7 \mathrm{ml}$ tube, Ref 05.1553 , Sarstedt) was transported to the hospital laboratory for routine analysis. The other sample $(1 \mathrm{ml} \mathrm{BD}$ Plastipak syringe, Ref 303172, Becton Dickinson, San Agustin del Guadalix) was immediately tested on the PoC device within the CT scan suite.

\section{Test methods}

Laboratory serum samples were processed using Roche Modular IDMS calibrated enzymatic creatinine analysis on a Cobas8000 platform (Roche, Inc.). The CE-marked PoC analyser was the i-STAT (Abbott Laboratories), a handheld device in current use in UK imaging departments [14]. The device utilises an analytic cartridge system (i-STAT Crea, whole blood sample size $65 \mu \mathrm{l}$ ) to reduce the risk of sample contamination. To avoid inter-device variation, a single analyser was used for the duration of the study at each site. To ensure the PoC device operated within the limits of acceptability for imprecision, daily quality assurance was performed during the research using the manufacturers' quality control (QC) materials at two levels of creatinine concentration. Staff training for PoC device operation was provided and competency assessed through collaboration with the local PoC management team, the pathology department and manufacturer representative.

\section{Blood test results management}

Following cannulation and $\mathrm{PoC}$ testing patients proceeded straight to scan with $\mathrm{CT}$ contrast injection as appropriate for their examination. The exception being where the PoC test result identified a decline in kidney function from their baseline result, which prompted a requirement to wait for laboratory confirmation (detailed in the participant information sheet and consent procedure).

The laboratory generated SCr and eGFR result were accessed from the hospital order communication system. The Abbot i-STAT does not provide an eGFR calculation and therefore, for consistency, both laboratory and PoC eGFR values were derived from the creatinine measurements using the Chronic Kidney Disease Epidemiology Collaboration (CKD-EPI) equation [30].

\section{Statistical Analysis}


Data were transcribed into the EDGE research management system (University of Southampton) and exported to Microsoft Excel ${ }^{\circledast}$ for descriptive analysis.

Scan-day creatinine and eGFR measures and the associated risk category (high risk =eGFR $\leq 30$; Moderate=eGFR31-44; Low $\geq 45$ ) [13] were compared and the results were also reviewed against the pre-scan (baseline) kidney function test. The clinical concordance between the baseline and scan-day results was evaluated through a modified Clarke Error Grid Analysis, a scatter plot of laboratory overlaid with eGFR results categorised into clinically relevant areas.

Scan-day and follow up PoC $\mathrm{WBCr}$ measurements and the relevant laboratory $\mathrm{SCr}$ results were compared using Bland Altman analysis. Passing-Bablok regression was used to explore the presence of proportional and constant error (from the slope and intercept co-efficient, respectively). This approach does not assume that any measurement error in either the laboratory or PoC measurements is normally distributed. These were generated in R (The R Foundation) using the packages ' $m c r$ ' and 'BlandAltmanLeh'.

\section{RESULTS}

Following exclusions 300 participant samples were obtained for PoC and laboratory analysis (Figure 1). Of these, 102 patients voluntarily returned for a follow up blood test within the specified time frame. Additionally, a further 19 outpatients underwent a clinically required kidney function test through follow up clinics within one week of contrast administration.

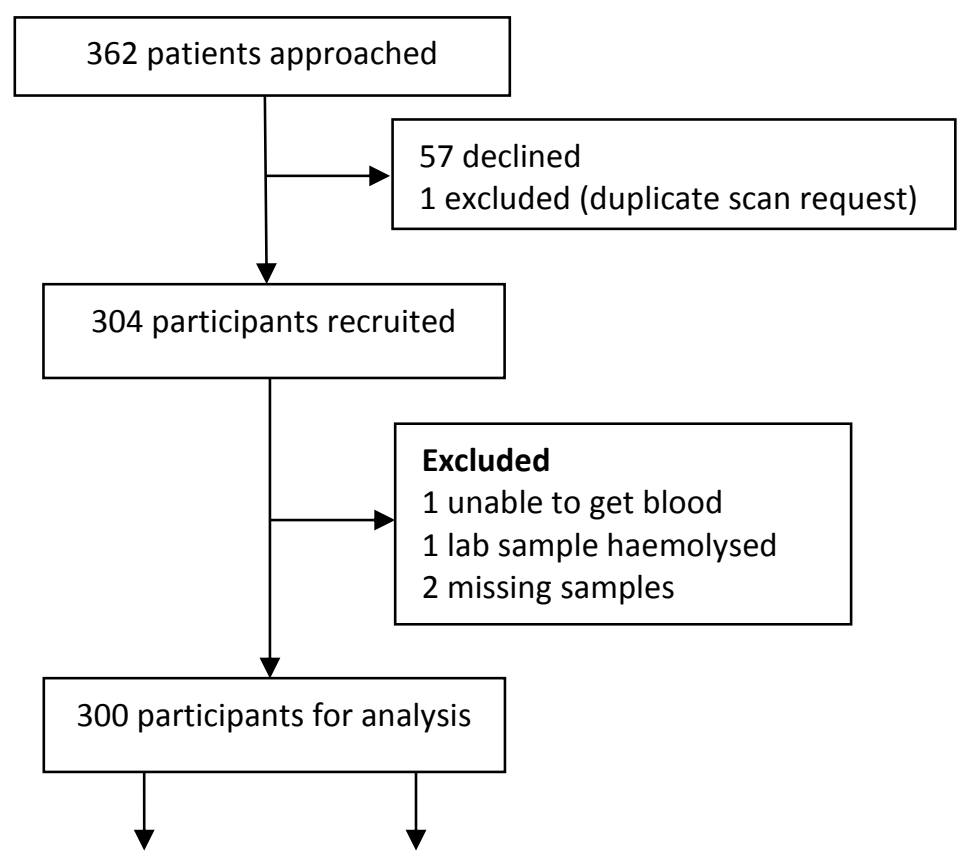




\section{Figure 1: Recruitment flow chart}

\section{Participant characteristics}

The sample comprised 155 males (51.6\%) and the mean age of participants was 65 years (range 23-91 years). The cohort was predominantly Caucasian with only 1 (0.3\%) Afro-Caribbean participant. The CT referrals originated from a number of different clinical specialities, including general surgery $(n=82$; $27.3 \%)$, urology $(n=52 ; 17.3 \%)$, oncology $(n=49 ; 16.3 \%)$ and respiratory medicine $(n=19 ; 6.3 \%)$. The majority of examinations related to the thorax and/or abdomen, with only $2(0.7 \%)$ examinations restricted to the head and/or neck.

\section{Baseline blood test availability}

Although baseline blood tests were expected to be carried out within the 3 months prior to the scan appointment the actual timing varied, with a mean of 3.7 weeks (range $0-59.6$ weeks), with $54.0 \%$ ( $n=162$ ) of patients tested on the day of referral for the scan. Importantly, $12.3 \%$ ( $n=37)$ of blood tests referrals had to be initiated by the radiology department to ensure compliance with local screening procedures and to enable the scan to be performed within clinical pathway timescales. Despite these measures, 4 patients still presented for their scan appointment with no recent kidney function results available. All consented to study participation with 3 patients waiting for the laboratory result, the other patient was eventually scanned without contrast, as they could not wait for the laboratory test result.

\section{Scan day kidney function}

Only 14 patients (4.7\%) had a scan-day laboratory eGFR below $45 \mathrm{~mL} / \mathrm{min} / 1.73 \mathrm{~m}^{2}$, with a single case meeting the high risk threshold of $30 \mathrm{~mL} / \mathrm{min} / 1.73 \mathrm{~m}^{2}$ (PoC test result $28 \mathrm{~mL} / \mathrm{min} / 1.73 \mathrm{~m}^{2}$ ). Importantly, this patient had a baseline laboratory eGFR of $70 \mathrm{~mL} / \mathrm{min} / 1.73 \mathrm{~m}^{2}$ performed seventeen days earlier but declared an acute illness in the preceding week on the screening questionnaire. 
The screening questionnaire identified all patients with an eGFR of $<45 \mathrm{~mL} / \mathrm{min} / 1.73 \mathrm{~m}$, although half of patients indicated at least 1 risk factor, with the most common being hypertension, followed by acute illness and diabetes (Figure 2). Due to the small number of high and moderate risk cases (eGFR $\leq 30$ or $<45 \mathrm{~mL} / \mathrm{min} / 1.73 \mathrm{~m}$ ) logistic regression was not performed.

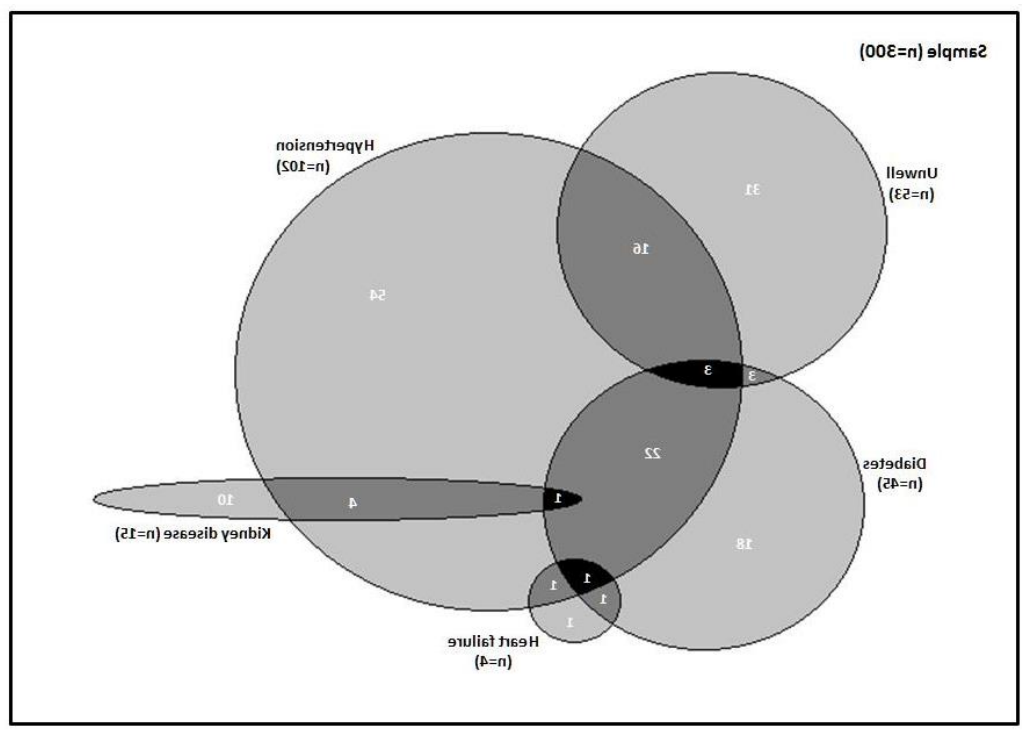

Figure 2. Risk factors (single and multiple) identified through the screening questionnaire

Comparison of the scan-day and baseline laboratory eGFR results confirmed that the majority of patients ( $n=281 / 300 ; 93.7 \%$ ) fell in the same risk category (Figure 3 and Table 1). For those 19 patients demonstrating a change in category, there was no significant difference in time interval between baseline and scan-day blood tests (whole cohort mean 29 days vs change 26 days; $p=0.888$ ).

In 8 cases an increased risk was identified, with the scan-day eGFR result suggesting a moderate (or high) risk of PC-AKI, rather than low risk. In 11 cases the risk was considered to have reduced, including one whose kidney function was noted to have improved from $38 \mathrm{~mL} / \mathrm{min} / 1.73 \mathrm{~m}$ to $64 \mathrm{~mL} / \mathrm{min} / 1.73 \mathrm{~m}$, supporting the decision to administer contrast. In one patient with known chronic kidney disease, the decision to withhold contrast was upheld despite a scan-day eGFR of $34 \mathrm{~mL} / \mathrm{min} / 1.73 \mathrm{~m}^{2}$ (PoC result $32 \mathrm{~mL} / \mathrm{min} / 1.73 \mathrm{~m}^{2}$ ), the previous eGFR result being $29 \mathrm{~mL} / \mathrm{min} / 1.73 \mathrm{~m}^{2}$ obtained five days earlier. 


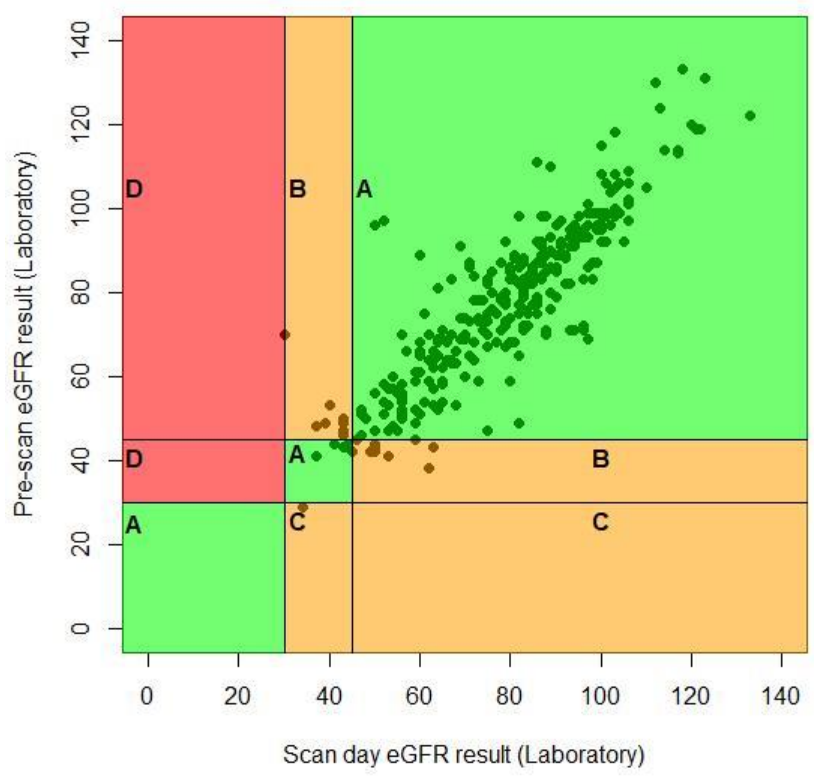

Figure 3. Error grid comparing baseline (pre-scan) eGFR laboratory result to scan-day eGFR laboratory result (see table 1 for key)

Table 1. Patient management implications of concordance between baseline (pre-scan) eGFR laboratory result and scan-day eGFR laboratory result

\begin{tabular}{|c|l|c|}
\hline Zone & \multicolumn{1}{|c|}{ Implication if management based on pre-scan eGFR result } & No (\%) \\
\hline A & Same risk category - appropriate management & 281 (93.7) \\
\hline B & $\begin{array}{l}\text { Changed from low risk to moderate risk, or vice versa. No impact on } \\
\text { management (if all patients orally pre-hydrated as standard). }\end{array}$ & 1 (5.7) \\
\hline C & $\begin{array}{l}\text { Changed from being at high risk to either moderate or low risk, } \\
\text { potential for unnecessary prophylaxis or withholding of contrast }\end{array}$ & $1(0.3)$ \\
\hline D & $\begin{array}{l}\text { Incorrect classification and potential for increased risk of PC-AKI due } \\
\text { to insufficient prophylaxis }\end{array}$ & 1 \\
\hline
\end{tabular}

\section{Follow up blood test results}

The characteristics of the patients attending for follow up blood tests were similar to those observed in the whole cohort, with no significant difference in gender $(p=0.644)$, age $(p=0.533)$ and scan-day clinical risk category $(p=0.602)$ based on laboratory eGFR result. Of the 121 patients who had a follow up blood test undertaken (study $(n=102)$ or clinically initiated $(n=19))$, no instances of PC-AKI were demonstrated. A small number $(n=5)$ of patients had an acute decline in kidney function, with $>25 \%$ increase from the initial PoC WBCr level (Table 2). All had been administered 95ml of contrast, no other common demographic factors were identified. A number of these patients acknowledged $(n=3)$ not following post-procedural hydration advice, no other attributable cause for the rise in creatinine was identified. 
Table 2. - Characteristics of the patients identified with an increased level (>25\%) of PoC whole blood creatinine $(\mathrm{WBCr})$ post contrast administration.

\begin{tabular}{|c|c|c|c|c|c|}
\hline $\begin{array}{l}\text { Age } \\
\text { (years)/ } \\
\text { gender }\end{array}$ & $\begin{array}{l}\text { Scan day } \\
\text { WBCr } \\
\text { (eGFR) }\end{array}$ & $\begin{array}{l}\text { Follow up } \\
\text { WBCr } \\
\text { (eGFR) }\end{array}$ & $\begin{array}{l}\text { \% change } \\
\text { in WBCr }\end{array}$ & $\begin{array}{l}\text { Interval } \\
\text { (hrs) }\end{array}$ & $\begin{array}{l}\text { Examination } \\
\text { performed }\end{array}$ \\
\hline $68 F^{*}$ & $75(71)$ & $101(49)$ & 34.7 & 48.5 & CT head, chest, liver \\
\hline $74 F^{*}$ & $101(47)$ & $132(34)$ & 30.7 & 49.5 & $\begin{array}{l}\text { CT neck, chest, } \\
\text { abdomen, pelvis }\end{array}$ \\
\hline $74 F^{*}$ & $82(62)$ & $107(45)$ & 30.5 & 75.5 & $\begin{array}{l}\text { CT colonography (inc. } \\
\text { CT abdomen, pelvis) }\end{array}$ \\
\hline $53 \mathrm{~F}$ & $41(113)$ & $55(103)$ & 34.1 & 72.5 & $\begin{array}{l}\text { CT Chest, abdomen, } \\
\text { pelvis }\end{array}$ \\
\hline $45 \mathrm{M}^{*}$ & $89(90)$ & $132(56)$ & 48.3 & 48.0 & CT urogram \\
\hline
\end{tabular}

Gender M=male; F=female

*Patients referred to Ambulatory Care Unit, advised hydration and return ( 3 at $24 \mathrm{hrs} ; 1$ at $24+48 \mathrm{hrs}$ ), kidney function improved on follow up bloods and patients discharged from hospital care.

\section{PoC performance}

The PoC analysis was performed by radiographers (clinical and/or research based) or support staff (Table 3), depending on staff availability.

Table 3: Roles of the individuals performing the point of care analysis and number of test failures

\begin{tabular}{|lllll|}
\hline Role & Scan day & Follow up & Total & $\begin{array}{l}\text { Number of } \\
\text { failures } \mathbf{n}(\%)\end{array}$ \\
\hline Clinical Support Worker $($ CSW) $(n=5)$ & 43 & 32 & 75 & $1(1.3)$ \\
Radiographer $(n=2)$ & 24 & 19 & 43 & $3(7.0)$ \\
Researcher $(n=3)$ & 233 & 51 & 284 & $4(1.4)$ \\
Total & $\mathbf{3 0 0}$ & $\mathbf{1 0 2}$ & $\mathbf{4 0 2}$ & $\mathbf{8 ( 2 . 0 )}$ \\
\hline
\end{tabular}

Six test failures were recorded on the scan day for the Abbott i-STAT device, of these 2 related to an insufficient sample volume, 2 test cartridges were overfilled and 2 were due to an error with the cartridge itself. In relation to the follow-up tests, 2 failures were recorded, both due to the test cartridge being overfilled. All tests were successful on the second attempt.

All QC tests fell within the limits set by the manufacturer.

\section{Concordance between the i-STAT and laboratory measures}

The average (mean) bias of the PoC device in relation to the laboratory standard was close to zero (Table 4 and Fig 4). The constant error between the Abbott i-STAT PoC scan-day measurements and the laboratory scan-day measurements was $-3.71(95 \% \mathrm{Cl}:-6.41$ to -0.50$)$ and the proportional error was 1.036 (95\% Cl: 1.00 to 1.07$)$. 
Table 4. Analysis for the Abbott i-STAT PoC device compared to the laboratory reference standard on scan day and at follow up

\begin{tabular}{|c|c|c|c|}
\hline & $\begin{array}{c}\text { Average Bias } \\
\qquad(95 \% \mathrm{Cl})\end{array}$ & $\begin{array}{c}\text { 95\% Limits of } \\
\text { Agreement }(95 \% \mathrm{CI})\end{array}$ & $\begin{array}{c}\text { Correlation } \\
\text { Coefficient } \\
(95 \% \mathrm{Cl})\end{array}$ \\
\hline Scan-day $(n=300)$ & $\begin{array}{c}-0.21 \\
(-1.01 \text { to } 0.58)\end{array}$ & $\begin{array}{c}-13.94(-15.32 \text { to }-12.56) \\
\text { to } \\
13.51(12.14 \text { to } 14.89)\end{array}$ & $\begin{array}{c}0.948 \\
(0.935 \text { to } 0.958)\end{array}$ \\
\hline Follow up ( $n=102$ ) & $\begin{array}{c}-0.59 \\
(-1.81 \text { to } 0.63)\end{array}$ & $\begin{array}{c}-12.77(-14.88 \text { to }-10.65) \\
\text { to } \\
11.59(9.48 \text { to } 13.70)\end{array}$ & $\begin{array}{c}0.949 \\
(0.926 \text { to } 0.966)\end{array}$ \\
\hline
\end{tabular}

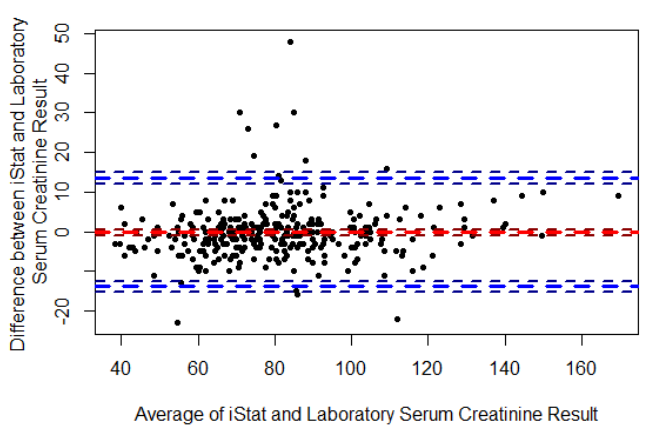

Figures 4A \& B: Bland-Altman plots demonstrating the average bias of the Abbott i-STAT compared to laboratory measured serum creatinine measured on scan day (4A) and follow up (4B), including $95 \%$ levels of agreement.

\section{DISCUSSION}

Kidney function testing prior to intravascular contrast administration is only advocated for patients with identified risk factors [9,12-14]. However, the limited clinical history available to radiology, activity volumes and complex

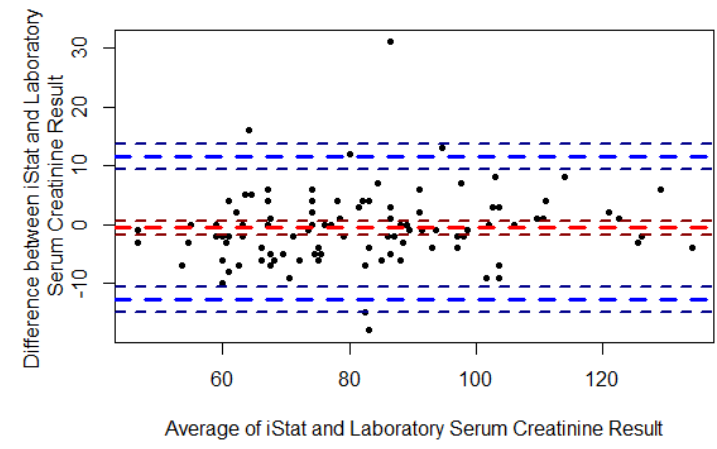
workflows mean pre-scan assessment of eGFR is the most common risk assessment technique $[1,15,31]$. Importantly, for centres which mandate a local pre-scan blood test screening procedure, a patient presenting without a current eGFR result may need to be rebooked, reducing scanner capacity and more importantly introducing a further delay to diagnosis and treatment. 
There is no consensus on the optimal time interval between eGFR result and contrast media administration for stable outpatients; rather this should, in practice, be guided by clinical judgement based on the likelihood that kidney function has deteriorated significantly [25]. Patient safety remains of paramount concern and our results suggest that for a small number of patients a blood test result obtained in the weeks or months prior to contrast administration is not reliable. An appropriate decision may only be possible with scan-day screening and kidney function assessment; the implications of not considering a potential change in eGFR has already been highlighted in a UK Coroner's inquest [32]. Our study has confirmed the validity of such a screening questionnaire to identify acute illness and co-morbidities linked to the increased risk of PC-AKI, similar to previous studies [23,26-29]. The high sensitivity, but relatively poor specificity of the tool to identify the small number of patients with radiology relevant kidney dysfunction is likely a factor of the broad questions used during this research study, and supports further modification to the questionnaire. Although screening promotes the identification of risk factors, without access to immediate blood test results this information is limited in its usefulness [26]. The results do confirm the potential for reducing inappropriate testing of patients with no risk factors.

There remains controversy around the incidence of PC-AKI. However, it is widely acknowledged that the greatest risk is in patients with an eGFR $\leq 30 \mathrm{~mL} / \mathrm{min} / 1.73 \mathrm{~m}^{2}$, with $45 \mathrm{~mL} / \mathrm{min} / 1.73 \mathrm{~m}^{2}$ consistently cited as a level above which the risk is very low or non-existent $[4,11,13,14,33]$. In our study there were no instances of PC-AKI, with only $5 \%$ demonstrating a rise in $\mathrm{WBCr}$ of greater than $25 \%$. It is key to note that this small cohort of patients was not identified as high risk in pre-procedure screening, and although dehydration during the post-scan period was identified as an anecdotal contributor, it cannot be confirmed. This raises further questions as to whether centres are, or should be, advocating the maintenance of fluid intake, particularly post procedure. Although there is no strong evidence for the use of peri-procedural oral hydration in patients with a moderate risk of PC-AKI, this remains a common strategy in practice [15]. For centres where scan-day risk screening is in place and who opt for an oral hydration strategy for certain risk categories, it may be easier to provide hydration advice to all patients, where there are no contra-indications. Hydration prior to their CT scan appointment could avoid insufficient prophylaxis, or delays, at the appointment. In the absence of evidence, this may not be the most cost-effective approach, but does enable efficient workflow and maintenance of patient safety.

The concordance of the study PoC device with the laboratory gold standard is consistent with other evaluations $[16,17,23,24,34]$ and provides reassurance around the test results. Following appropriate training radiographers and support staff were able to undertake sample testing with a relatively low 
failure rate, which appears to reduce with experience. These outcomes confirm that it is feasible to utilise a PoC device for kidney function screening in a radiology setting.

\section{Strengths}

This study has explored the clinical utility of a screening questionnaire in combination with PoC for risk stratification and evaluation of kidney function prior to intravenous contrast media administration. Additionally, monitoring for AKI post contrast administration (follow up) was offered to all participants and not restricted to patients with a baseline (pre-scan) reduced kidney function, a criticism of previous research.

\section{Limitations}

The low number of patients with pre-existing kidney dysfunction is in line with previous studies $[17,26]$ and the stable out-patient population. This does however reinforce the need for a re-examination of the clinical pathway based on over-testing of patients. The study was limited to the review of a single PoC device in relation to test performance and failures, no other assessment of the practicalities associated with PoC use in clinical practice was evaluated. In this study PoC testing utilised a single portable hand-held device however, a range of alternatives are available on the market.

The need for efficient workflow and rapid turn-around of referrals for contrast-enhanced imaging studies supports the introduction of PoC creatinine testing. However, due to previous concerns around the accuracy of the PoC creatinine technology, it is yet to make its way into mainstream use. This study has demonstrated that PoC creatinine testing is feasible within CT clinical pathways. Further evidence is required of the feasibility and practicality of embedding PoC technology into clinical practice through multi-centre evaluation on a much larger population.

\section{Conflict of interest}

The authors have no conflicts of interest to declare

\section{Funding}

The work received funding from the National Cancer Diagnostics Capacity Fund, NHS England. Andrew Lewington, Bethany Shinkins and Michael Messenger are currently supported by the NIHR MIC- Leeds In Vitro Diagnostics Co-operative (was previously NIHR Leeds Diagnostic Evidence Co- 
operative). The views and opinions expressed therein are those of the authors and do not necessarily reflect those of the HTA programme, NIHR, NHS or the Department of Health. The authors accept full responsibility for the research.

\section{Role of funding source}

The funding agency had no role in: the design and conduct of the study; collection, management, analysis, and interpretation of the data; preparation, review, or approval of the manuscript; nor decision to submit the manuscript for publication.

\section{ACKNOWLEDGEMENTS}

We are grateful to the radiographers and support staff who enabled the research to be carried out in the clinical setting. We also acknowledge the support of Mid Yorkshire Hospitals Research and Development team and the Pathology Department, in particular Paul Walker and the PoC management team (Tracey Eastwood, Ebrahim Rawat and colleagues) who provided equipment oversight and advice.

\section{REFERENCES}

1. Bargnoux, A-S., Beaufils, O., Oguike, M., et al., Point-of-care creatinine testing in patients receiving contrast-enhanced computed tomography scan, Clin Chim Acta 478 (2018) 111-3.

2. McDonald, R.J., McDonald, J.S., Carter, R.E., et al, Intravenous contrast material exposure is not an independent risk factor for dialysis or mortality, Radiology 273 (2014) 714-25.

3. McDonald, R.J., McDonald, J.S., Bida, J.P., et al, Intravenous contrast material-induced nephropathy causal or coincident phenomenon? Radiology 267 (2013) 106-18.

4. McDonald, J.S., McDonald, R.J., Carter, R.E., Katzberg, R.W., Kallmes, D.F., Williamson, E.E. Risk of intravenous contrast material mediated acute kidney injury: a propensity score-matched study stratified by baseline estimated glomerular filtration rate, Radiology 271 (2014) 65-73.

5. McDonald, J.S., McDonald, R.J., Comin, J., et al, Frequency of acute kidney injury following intravenous contrast medium administration: a systematic review and meta-analysis, Radiology 267 (2013) 119-128.

6. Wilhelm-Leen, E., Montez-Rath, M.E., Chertow, G., Estimating the Risk of RadiocontrastAssociated Nephropathy, J Am Soc Nephrol 28 (2017) 653-9.

7. Hinson, J.S., Ehmann, M.R., Fine, D.M., et al., Risk of acute kidney injury after intravenous contrast media administration, Ann Emerg Med 69 (2017) 577-86.

8. Aycock, R.D., Westafer, L.M., Boxen, J.L., Majlesi, N., Schoenfeld, E.M., Bannuru, R.R., Acute kidney injury after computed tomography: a meta-analysis, Ann Emerg Med 71 (2017) 44-53. 
9. European Society of Urogenital Radiology. ESUR guidelines on contrast media, v10.0. http://www.esur-cm.org/index.php/en/, 2018 (accessed 7 August 2018).

10. Mitchell, A.M., Kline, J.A., Jones, A.E., Tumlin, J.A., Major adverse events one year after acute kidney injury after contrast-enhanced computed tomography, Ann Emerg Med 66 (2015) 267-74.

11. Davenport, M.S., Khalatbari, S., Cohan, R.H., Dillman, J.R., Myles, J.D., Ellis, J.H., Contrast material-induced nephrotoxicity and intravenous low-osmolality iodinated contrast material: risk stratification by using estimated glomerular filtration rate, Radiology 268 (2013) 719-28.

12. American College of Radiologists. ACR manual on contrast media, v10.3. https://www.acr.org//media/ACR/Files/Clinical-Resources/Contrast_Media.pdf , 2018 (accessed 7 August 2018)

13. Royal Australian \& New Zealand College of Radiologists. lodinated contrast media guideline. https://www.ranzcr.com/documents/573-iodinated-contrast-guidelines-2016/file , 2016 (accessed 7 August 2018)

14. Canadian Association of Radiologists. Consensus Guidelines for the prevention of contrast induced nephropathy, https://car.ca/wp-content/uploads/Prevention-of-Contrast-InducedNephropathy-2011.pdf , 2011 (accessed 7 August 2018)

15. Harris, M.A., Snaith, B., Clarke, R., Strategies for assessing renal function prior to outpatient contrast-enhanced CT: a UK survey, Br J Radiol 89 (2016) 20160077. https://doi.org/10.1259/bjr.20160077.

16. Lee-Lewandrowski, E., Chang, C., Gregory, K., Lewandrowski, K., Evaluation of rapid point-of-care creatinine testing in the radiology service of a large academic medical center: Impact on clinical operations and patient deposition, Clin Chim Acta 413 (2012) 88-92.

17. Korpi-Steiner, N.L., Williamson, E.E., Karon, B.S., Comparison of three whole blood creatinine methods for estimation of glomerular filtration rate before radiographic contrast administration, Am J Clin Pathol 132 (2009) 920-926.

18. Dimeski, G., Tilley, V., Jones, B.W., Brown, N.N., Which point-of-care analyser for radiology: direct comparison of the i-Stat and StatStrip creatinine methods with different sample types, Ann Clin Biochem 50 (2013) 47-52.

19. Haneder, S., Gutfleisch, A., Meier, C., et al., Evaluation of a handheld creatinine device for realtime determination of serum creatinine in radiology departments, World J Radiol 4 (2012) 328334.

20. Morita, S., Suzuki, K., Masukawa, A., Ueno, E., Assessing renal function with a rapid, handy, point-of-care whole blood creatinine meter before using contrast materials, Jpn J Radiol 29 (2011) 187-193.

21. Aumatell, A., Sharpe, D., Reed, W., Validation of the Nova StatSensor Creatinine for testing blood before contrast computed tomography studies, Point of Care 9 (2010) 25-31.

22. Inoue, A., Nitta, N., Ohta, S., et al., StatSensor-I point-of-care creatinine analyser may identify patients at high-risk of contrast-induced nephropathy, Exp Ther Med 13 (2017) 3505-3508.

23. Too, C.W., Ng, W.Y., Tan, C.C., Mahmood, M.I., Tay, K.H., Screening for impaired renal function in outpatients before iodinated contrast injection: comparing the Choyke questionnaire with a rapid point-of-care-test, Eur J Radiol 84 (2015) 1227-1231.

24. Snaith, B., Harris, M.A., Shinkins, B., Jordaan, M., Messenger, M., Lewington, A., Point-of-care creatinine testing for kidney function measurement prior to contrast-enhanced diagnostic imaging: evaluation of the performance of three systems for clinical utility, Clin Chem Lab Med 56 (2018) 1269-76. DOI: 10.1515/cclm-2018-0128 
25. National Institute for Health and Care Excellence. Point-of-care creatinine tests before contrast enhanced imaging. Medtech innovation briefing [MIB136].

https://www.nice.org.uk/advice/mib136/resources/pointofcare-creatinine-tests-beforecontrastenhanced-imaging-pdf-2285963399057605, 2018 (accessed 7 August 2018)

26. Zähringer, C., Potthast, S., Tyndall, A.J., Bongartz, G., Hohmann, J., Serum creatinine measurements: evaluation of a questionnaire according to the ESUR guidelines, Acta Radiol 56 (2015) 628-34.

27. Choyke, P.L., Cady, J., DePollar, S.L., Austin, H., Determination of serum creatinine prior to iodinated contrast media: is it necessary in all patients? Tech Urol 4 (1998) 65-69.

28. Ledermann, H.P., Mengiardi, B., Schmid, A., Froehlich, J.M., Screening for renal insufficiency following ESUR (European Society of Urogenital Radiology) guidelines with on-site creatinine measurements in an outpatient setting, Eur Radiol 20 (2010) 1926-1933.

29. Azzouz, M., Rømsing, J., Thomsen, H.S., Can a structured questionnaire identify patients with reduced renal function? Eur Radiol 24 (2014) 780-4.

30. CKD-EPI Calculator for Adults (SI Units). National Institute of Diabetes and Digestive and Kidney Diseases. https://www.niddk.nih.gov/health-information/communicationprograms/nkdep/laboratory-evaluation/glomerular-filtration-rate-calculators/ckd-epi-adults-siunits (accessed 7 August 2018)

31. van der Molen, A.J., Reimer, P., Dekkers, I.A., et al., Post-contrast acute kidney injury. Part 2: risk stratification, role of hydration and other prophylactic measures, patients taking metformin and chronic dialysis patients : Recommendations for updated ESUR Contrast Medium Safety Committee guidelines, Eur Radiol 28 (2018) 2856-69. DOI: 10.1007/s00330-017-5247-4

32. Courts and Tribunals Judiciary, Bryan Whitby [online], https://www.judiciary.gov.uk/publications/bryan-whitby/ (accessed 7 August 2018)

33. van der Molen, A.J., Reimer, P., Dekkers, I.A., et al., Post-contrast acute kidney injury - Part 1:Definition, clinical features, incidence, role of contrast medium and risk factors, Eur Radiol 28 (2018) 2845-55. DOI: 10.1007/s00330-017-5246-5.

34. You, J.S., Chung, Y.E., Park, J.W., et al., The usefulness of a rapid point-of-care creatinine testing for the prevention of contrast-induced nephropathy in the emergency department, Emerg Med J 30 (2013) 555-58. DOI: 10.1136/emermed-2012-201285 